Original Research Paper

\title{
Some Proposed Solutions to Achieve Nuclear Fusion
}

\author{
${ }^{1}$ Relly Victoria Virgil Petrescu, ${ }^{2}$ Aversa Raffaella, ${ }^{3}$ Samuel Kozaitis, \\ ${ }^{2}$ Apicella Antonio and ${ }^{1}$ Florian Ion Tiberiu Petrescu \\ IIFToMM, ARoTMM, Bucharest Polytechnic University, Bucharest, Romania \\ ${ }^{2}$ Advanced Material Lab, Department of Architecture and Industrial Design, \\ Second University of Naples, 81031 Aversa (CE), Italy \\ ${ }^{3}$ Florida Institute of Technology, USA
}

Article history

Received: 06-07-2017

Revised: 13-07-2017

Accepted: 19-07-2017

Corresponding Author: Florian Ion Tiberiu Petrescu

IFToMM, ARoTMM,

Bucharest Polytechnic

University, Bucharest,

Romania

Email: scipub02@gmail.com

\begin{abstract}
Despite research carried out around the world since the 1950s, no industrial application of fusion to energy production has yet succeeded, apart from nuclear weapons with the H-bomb, since this application does not aims at containing and controlling the reaction produced. There are, however, some other less mediated uses, such as neutron generators. The fusion of light nuclei releases enormous amounts of energy from the attraction between the nucleons due to the strong interaction (nuclear binding energy). Fusion it is with nuclear fission one of the two main types of nuclear reactions applied. The mass of the new atom obtained by the fusion is less than the sum of the masses of the two light atoms. In the process of fusion, part of the mass is transformed into energy in its simplest form: Heat. This loss is explained by the Einstein known formula $\mathrm{E}=\mathrm{mc}^{2}$. Unlike nuclear fission, the fusion products themselves (mainly helium 4) are not radioactive, but when the reaction is used to emit fast neutrons, they can transform the nuclei that capture them into isotopes that some of them can be radioactive. In order to be able to start and to be maintained with the success the nuclear fusion reactions, it is first necessary to know all this reactions very well. This means that it is necessary to know both the main reactions that may take place in a nuclear reactor and their sense and effects. The main aim is to choose and coupling the most convenient reactions, forcing by technical means for their production in the reactor. Taking into account that there are a multitude of possible variants, it is necessary to consider in advance the solutions that we consider them optimal. The paper takes into account both variants of nuclear fusion and cold and hot. For each variant will be mentioned the minimum necessary specifications.
\end{abstract}

Keywords: Nuclear Fusion, Cold Nuclear Fusion, Thermonuclear Reaction, Possible Nuclear Reactions, Nuclear Power, Some Fusion Solutions, Dynamic Synthesis, Forces, Velocities, Powers, Dimensions

\section{Introduction}

Nuclear fusion, sometimes referred to as thermonuclear fusion, is a process where two light atomic nuclei come together to form a heavier nucleus. This reaction is at work in a natural way in the Sun and most stars of the Universe.

The fusion of light nuclei releases enormous amounts of energy from the attraction between the nucleons due to the strong interaction (nuclear binding energy). Fusion it is with nuclear fission one of the two main types of nuclear reactions applied (Shultis and Faw, 2002).
The mass of the new atom obtained by the fusion is less than the sum of the masses of the two light atoms. In the process of fusion, part of the mass is transformed into energy in its simplest form: heat. This loss is explained by the Einstein known formula $\mathrm{E}=\mathrm{mc}^{2}$.

One of its interests is to be able to obtain theoretically much more energy: First to mass of "fuel" equal, the fusion releases three to four times more energy than fission. Then, the "fuel" stock is much larger: The oceans naturally contain such a mass of deuterium (33 $\mathrm{g} / \mathrm{m}^{3}$ ) that they could theoretically satisfy the current 
energy consumption of the human species for one hundred million Years $\left(1 \mathrm{~m}^{3}\right.$ of water can potentially provide as much energy as the combustion of $700 \mathrm{t}$ of oil).

Despite research carried out around the world since the 1950s, no industrial application of fusion to energy production has yet succeeded, apart from nuclear weapons with the H-bomb, since this application does not Aims at containing and controlling the reaction produced. There are, however, some other less mediated uses, such as neutron generators (Thermonuclear weapon, from Wikipedia).

Unlike nuclear fission, the fusion products themselves (mainly helium 4) are not radioactive, but when the reaction is used to emit fast neutrons, they can transform the nuclei that capture them into isotopes that some of them can be radioactive.

We must not confuse nuclear fusion with the fusion of the core of a nuclear reactor, which is a particularly formidable nuclear accident.

The only nuclear reaction carried out on an industrial scale up to now is that of nuclear fission. With all that there were some nuclear accidents and the raw material used as fuel and residue also are radioactive, energy from nuclear fission represented for humanity a necessary evil. She managed to prevent an energy major crisis for humanity.

We were not allowed back in the caves as it has started with millions of years ago. We do not want to give up nor to our current homes comfortable, that we make them to be warm in winter and cooling them in summer you (to feel us better).

The energy for fission helped us to avoid a crisis disaster and in addition to prolong the life of the hydrocarbons. Today we are a little better.

The advanced technologies allowed us to implement new renewable energies, sustainable, green and friendly for man and to the environment. It is better to continue to implement other and other central solar energy, wind or hydro. But if we have a moment of rest, it does not mean that we must give up to a beautiful dream of humanity, namely the one to bring the sun on the Earth. In other words, we must continue to try to devise and achieve the nuclear fusion, industrial and peaceful (Halliday and Robert, 1966). A let us begin by presenting a wellknown diagram among specialists (Fig. 1).

Stable nuclei are represented by filled circles (blackened). Radioactive nuclei (i.e., those which spontaneously decay, emitting electrons or alpha particles) are represented by unfilled circles.

On the $\mathrm{x}$-axis was represented atomic number $\mathrm{Z}$ (indicate number of protons in the nucleus).

On the y-axis was represented the number of the neutronic $\mathrm{N}$ (which indicate number of neutrons in the nucleus; Progress in Fusion, ITER).

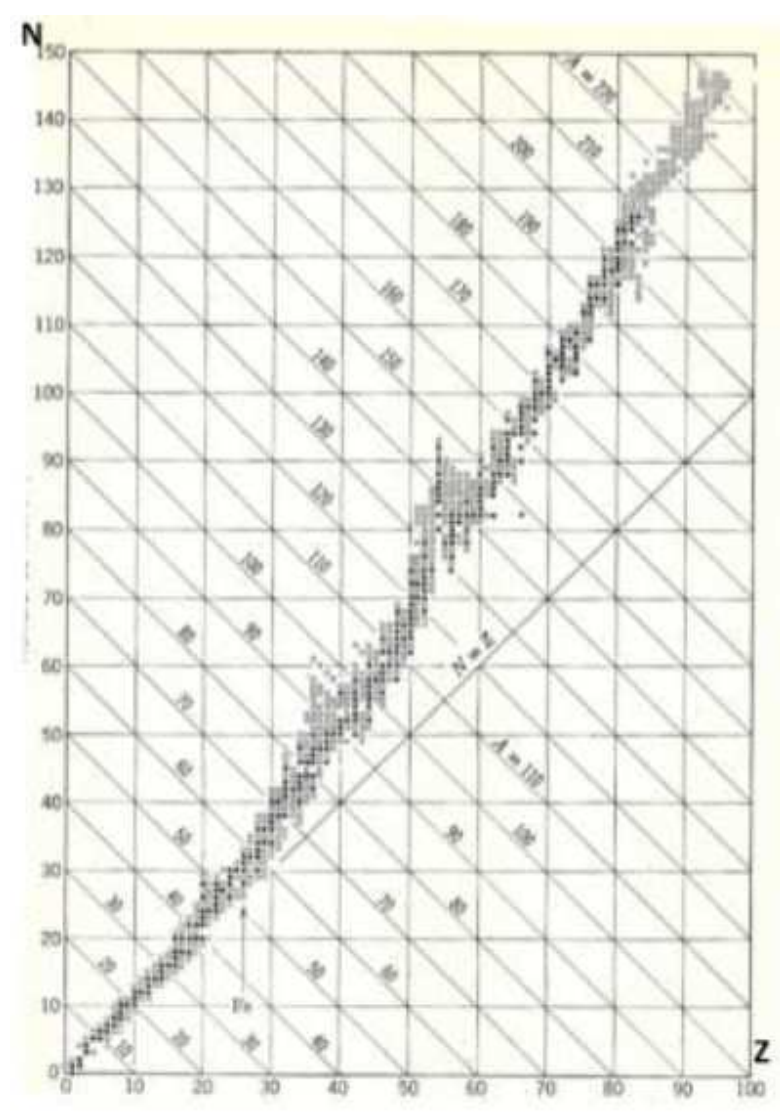

Fig. 1. Diagram of atomic cores (atomic nuclei)

It is important to note that almost every known element from the Mendeleev's table of chemical exists in several forms, in reality, called isotopes. To understand better what are isotopes of an element, consider for example iron (to follow the arrow that indicates iron isotopes), which has 26 permanent protons $(Z=26)$, but it may have in its various isotopes (eight in number) various structures neutron $(\mathrm{N}=26-33)$. The iron has four isotopes stable and four radioactive.

The next logical step is to try to determine the approximate Coulomb force acting between two protons in the atom of iron.

\section{Determining of the Approximate Coulomb Force Acting between two Protons in the Atom of Iron}

One uses the known relationship I:

$F_{c}=\frac{1}{4 \pi \varepsilon_{0}} \cdot \frac{q_{1} \cdot q_{2}}{r^{2}}$

Because it may be interesting to know and the gravitational force acting between two close protons, is 
good to determine it as well using expression II extremely popular:

$$
F_{g}=G \cdot \frac{m_{1} \cdot m_{2}}{r^{2}}
$$

It uses the beginning, static approximate radius of a proton $\left(r_{p s}=4 \mathrm{E}-15[\mathrm{~m}]\right)$ :

$$
F_{c s}=14.41605963[N] \text { and } F_{g s}=1.17152 E-35[N]
$$

Then we determine the same forces for two accelerated protons to the velocity $v=691665[\mathrm{~m} / \mathrm{s}]$, when a radius of a dynamic proton is $\left(r_{p d}=3.83566 \mathrm{E}-19[\mathrm{~m}]\right)$ :

$$
F_{c d}=1567781889[N] \text { and } F_{g d}=1.27406 E-27[N]
$$

At the subatomic scale such (Coulomb) forces are huge. $14 N$ is a huge force for a proton particle. Gravitational forces acting between two close protons are negligible. "Who speaks for the influence of gravity, at quantum and sub-quantum levels, let leave us (still need manpower in agriculture, because we are already increasingly more)".

If the static Coulomb force between two protons (14 $\mathrm{N})$ is so great, what to say about its huge value $(1.5 \mathrm{E}+9$ $\mathrm{N})$ when the protons are accelerated at a high speed, $v=$ $691665[\mathrm{~m} / \mathrm{s}]$ ?

Huge separation forces between protons of a nucleus are equilibrated by the nuclear forces and by the increased number of neutrons compared to existing protons in the nucleus.

At a certain level of acceleration of particles when they reach a speed limit, Coulomb forces are so great as they to exceed the nuclear forces of attraction.

At that moment nuclei break in their components or in other elements (or isotopes).

Also on this energy level are possible mergers but only to create lighter atoms.

Basically this is the underlying mechanism that can produce fusion.

Free, such a level of acceleration of nuclear particles, can be obtained only by making huge temperatures, as happens in stars (warm fusion, or thermonuclear reaction). It takes for that of about 40 trillion degrees.

An easier way to accomplish this stage, is to accelerating particles using particle accelerators (preferably circular accelerators).

The manner acceleration can be used to achieve the cold fusion and also in the hot modified fusion (a warm fusion, to a high temperature but not as in sun, helped by particle accelerator).

The work proposes these two means of production of the reaction of merger controlled on an industrial scale.

\section{Remark}

Dynamic radius has been determined with a relationship original (III), (Petrescu and Calautit, 2016a):

$$
R=\sqrt{\frac{5}{4} \cdot \frac{h \cdot \sqrt{c^{2}-v^{2}} \cdot \sqrt{c^{2}-\frac{v^{2}}{2}-c \cdot \sqrt{c^{2}-v^{2}}}}{\pi \cdot m_{0} \cdot c^{2} \cdot v}}
$$

If gravitational forces acting between two close protons are negligible, which are the forces at these levels (atomic or quantum level and subatomic, nuclear or sub-quantum level), (Reid, 1968)?

Known are four forces:

- Inertial forces

- Electric forces

- Magnetic forces

- Nuclear forces, strong and weak

What is the nature of nuclear forces? They almost always manage to balance the electrical forces. In a static nucleus, nuclear forces amount to the high value type Coulomb forces, of about $14 \mathrm{~N}$. If they are moving nucleons, the enormous increase of electrical forces is permanent chased (followed) by the nuclear forces, which are also increasing. When the distance between two nucleons becomes about E-19 [m], Coulomb and Nuclear forces take both a huge value of about $1.5 \mathrm{E}+9$ [N]. This clearly indicates that the nature of nuclear forces is similar to the electrical or electromagnetic forces (Petrescu and Calautit, 2016b).

\section{Results and Discussion}

First time Deuterium was extracted from water in 1931 by Harold Urey (Harold Urey, from Wikipedia). Small linear electrostatic accelerators have indicated that D-D reaction (fusion of two deuterium nuclei) is exothermic, even at that time.

It is known that not only the second isotope of hydrogen (Deuterium) can produce fusion nuclear energy, but and the third (heavy) isotope of hydrogen (Tritium) may produce energy through a nuclear fusion.

First nuclear fusion reaction it is possible between two nuclei of Deuterium and may be obtained: One Tritium nucleus plus a proton and energy, either a helium isotope with a neutron and energy (Equation 1 and 2), (Petrescu, 2012; Petrescu and Calautit, 2016a; Petrescu and Petrescu, 2012; Petrescu et al., 2016):

$$
\begin{aligned}
& { }_{1}^{2} \mathrm{D}+{ }_{1}^{2} \mathrm{D} \rightarrow{ }_{1}^{3} \mathrm{~T}+1.01 \mathrm{MeV}+{ }_{1}^{1} \mathrm{H}+3.02 \mathrm{MeV} \\
& ={ }_{1}^{3} \mathrm{~T}+{ }_{1}^{1} \mathrm{H}+4.03 \mathrm{MeV}
\end{aligned}
$$




$$
\begin{aligned}
& { }_{1}^{2} \mathrm{D}+{ }_{1}^{2} \mathrm{D} \rightarrow{ }_{2}^{3} \mathrm{He}+0.82 \mathrm{MeV}+{ }^{1} n+2.45 \mathrm{MeV} \\
& ={ }_{2}^{3} \mathrm{He}+{ }^{1} n+3.27 \mathrm{MeV}
\end{aligned}
$$

Fusion reaction may occur and between a nucleus of Deuterium and one of Tritium (Equation 3) and this fusion nuclear reaction may be produced more easily than one between two deuterons (Equation 1 and 2) (Krane, 1987):

$$
\begin{aligned}
& { }_{1}^{2} \mathrm{D}+{ }_{1}^{3} \mathrm{~T} \rightarrow{ }_{2}^{4} \mathrm{He}+3.5 \mathrm{MeV}+{ }^{1} n+14.1 \mathrm{MeV} \\
& ={ }_{2}^{4} \mathrm{He}+{ }^{1} n+17.6 \mathrm{MeV}
\end{aligned}
$$

An important nuclear reaction may be produced between a nucleus of Deuterium and an isotope of Helium (Equation 4):

$$
\begin{aligned}
& { }_{1}^{2} \mathrm{D}+{ }_{2}^{3} \mathrm{He} \rightarrow{ }_{2}^{4} \mathrm{He}+3.6 \mathrm{MeV}+{ }_{1}^{1} \mathrm{H}+14.7 \mathrm{MeV} \\
& ={ }_{2}^{4} \mathrm{He}+{ }_{1}^{1} \mathrm{H}+18.3 \mathrm{MeV}
\end{aligned}
$$

The isotope of helium ${ }_{2}^{3} \mathrm{He}$ is obtained in the reaction of 2 .

The reaction of the 1 generates Tritium which together with Deuterium (if one of them or both have enough energy) produce the nuclear reaction of 3 to generate a lot of energy and helium, a non toxic, inert and very stable gas (Moses et al., 2009).

For this reason the group of nuclear reactions of fusion is an advantageous one, friendly, pure, peacefully and inexpensive (Kramer, 2011).

Naturally the Tritium appears in the nuclear reactor, only when the reaction of 1 is produced, but one may obtains more Tritium from the nuclear reaction (Equation 5):

$$
{ }_{3}^{6} \mathrm{Li}+{ }^{1} n \rightarrow{ }_{1}^{3} \mathrm{~T}+{ }_{2}^{4} \mathrm{He}+4.784 \mathrm{MeV}
$$

The bars of lithium are easily entered or extracted in the nuclear reactor and by this mechanism can be controlled very simple and the fusion reaction speed at any time.

The reaction of the 5 can help much the reaction to the merger, by controlling its production. Lithium reserves in the earth's crust would permit the operation of melting plants for more than 1,000 years and those of the oceans could meet the needs for millions of years.

Neutrons necessary for producing the reaction 5 are generated even in the reactor in the framework of the reactions 2 and 3 (Petrescu, 2012; Petrescu and Calautit, 2016a; Petrescu and Petrescu, 2012; Petrescu et al., 2016).

Raw materials to achieve nuclear fusion are the deuterium and lithium, more exactly heavy water and bars of lithium. Result a lot of energy and helium.
The reaction can be controlled easily through various methods. The reaction of the merger no tends to "get out of control" such as to the fission (being difficult to start it and easy to stop it), (Shultis and Faw, 2002).

Equation 6 can generates extra energy and it is much easier to achieve on the Earth than other possibilities:

$$
{ }_{1}^{3} T+{ }_{1}^{3} T \rightarrow{ }_{2}^{4} \mathrm{He}+2 \cdot{ }^{1} n+11.3 \mathrm{MeV}
$$

In a nuclear reactor of this type, supplied with Deuterium, can take place and other nuclear reactions, of which the most important are (Equation 7-9):

$$
\begin{aligned}
& { }_{2}^{3} \mathrm{He}+{ }_{2}^{3} \mathrm{He} \rightarrow{ }_{2}^{4} \mathrm{He}+2 \cdot{ }_{1}^{1} \mathrm{H}+12.9 \mathrm{MeV} \\
& { }_{2}^{3} \mathrm{He}+{ }_{1}^{3} \mathrm{~T} \rightarrow{ }_{2}^{4} \mathrm{He}+{ }_{1}^{1} \mathrm{H}+{ }^{1} \mathrm{n}+12.1 \mathrm{MeV} \\
& { }_{2}^{3} \mathrm{He}+{ }_{1}^{3} \mathrm{~T} \rightarrow{ }_{2}^{4} \mathrm{He}+4.8 \mathrm{MeV}+{ }_{1}^{2} \mathrm{D}+9.5 \mathrm{MeV} \\
& ={ }_{2}^{4} \mathrm{He}+{ }_{1}^{2} \mathrm{D}+14.3 \mathrm{MeV}
\end{aligned}
$$

Lithium with deuterium can still generate four other important reactions (Equation 10-13):

$$
\begin{aligned}
& { }_{1}^{2} \mathrm{D}+{ }_{3}^{6} \mathrm{Li} \rightarrow 2 \cdot{ }_{2}^{4} \mathrm{He}+22.4 \mathrm{MeV} \\
& { }_{1}^{2} \mathrm{D}+{ }_{3}^{6} \mathrm{Li} \rightarrow{ }_{2}^{3} \mathrm{He}+{ }_{2}^{4} \mathrm{He}+{ }^{1} n+2.56 \mathrm{MeV} \\
& { }_{1}^{2} \mathrm{D}+{ }_{3}^{6} \mathrm{Li} \rightarrow{ }_{3}^{7} \mathrm{Li}+{ }_{1}^{1} \mathrm{H}+5.0 \mathrm{MeV} \\
& { }_{1}^{2} \mathrm{D}+{ }_{3}^{6} \mathrm{Li} \rightarrow{ }_{4}^{7} \mathrm{Be}+{ }^{1} \mathrm{n}+3.4 \mathrm{MeV}
\end{aligned}
$$

Lithium can react and with hydrogen $=$ Protium $(\mathrm{a}$ proton; Equation 14) or with an isotope of $\mathrm{He}$ (Equation 15):

$$
\begin{aligned}
& { }_{1}^{1} \mathrm{H}+{ }_{3}^{6} \mathrm{Li} \rightarrow{ }_{2}^{4} \mathrm{He}+1.7 \mathrm{MeV}+{ }_{2}^{3} \mathrm{He}+2.3 \mathrm{MeV} \\
& ={ }_{2}^{4} \mathrm{He}+{ }_{2}^{3} \mathrm{He}+4.0 \mathrm{MeV} \\
& { }_{2}^{3} \mathrm{He}+{ }_{3}^{6} \mathrm{Li} \rightarrow 2 \cdot{ }_{2}^{4} \mathrm{He}+{ }_{1}^{1} \mathrm{H}+16.9 \mathrm{MeV}
\end{aligned}
$$

It should also be mentioned separately an extremely exciting nuclear reaction (Equation 16) that may occur between the stable isotope of boron with 6 neutrons (boron has five protons) and the first isotope of hydrogen, Protium (Hydrogen, from Wikipedia).

The reaction between hydrogen and boron can be achieved more easily than others and can generate a large amount of energy plus the inert gas, He:

${ }_{1}^{1} \mathrm{H}+{ }_{5}^{11} \mathrm{~B} \rightarrow 3 \cdot{ }_{2}^{4} \mathrm{He}+8.7 \mathrm{MeV}$ 
If the reaction of merger Protium-Protium can be produced only in the stars (as the Protium is a very stable isotope) on the Earth we can try the easiest to achieve the merger Tritium-Tritium (Equation 6), as the Tritium is an isotope unstable (see diagram of the Fig. 1).

In the laboratory may be carried out and the reactions between Protium and Boron (Equation 16), or Protium and Lithium (Equation 14).

\section{Conclusion}

First time Deuterium was extracted from water in 1931 by Harold Urey. Small linear electrostatic accelerators have indicated that D-D reaction (fusion of two deuterium nuclei) is exothermic, even at that time.

It is known that not only the second isotope of hydrogen (Deuterium) can produce fusion nuclear energy, but and the third (heavy) isotope of hydrogen (Tritium) may produce energy through a nuclear fusion.

If the reaction of merger Protium-Protium can be produced only in the stars on the Earth we can try the easiest to achieve the merger Tritium-Tritium.

In the laboratory may be carried out and the reactions between Protium and Boron, or Protium and Lithium.

What is the nature of nuclear forces? They almost always manage to balance the electrical forces. In a static nucleus, nuclear forces amount to the high value type Coulomb forces, of about $14 \mathrm{~N}$. If they are moving nucleons, the enormous increase of electrical forces is permanent chased (followed) by the nuclear forces, which are also increasing. When the distance between two nucleons becomes about E-19 [m], Coulomb and Nuclear forces take both a huge value of about $1.5 \mathrm{E}+9[\mathrm{~N}]$. This clearly indicates that the nature of nuclear forces is similar to the electrical or electromagnetic forces.

Gravitational forces, acting between two atomic (atomic or quantum level) or subatomic (subatomic, or nuclear or sub-quantum level; nucleons level) particles, are negligible.

\section{Acknowledgement}

This text was acknowledged and appreciated by Dr. Veturia CHIROIU Honorific member of Technical Sciences Academy of Romania (ASTR) PhD.

\section{Funding Information}

Petrescu F.I., 2012, Cold nuclear fusion. Plasma Physics and Fusion Technology (S70), INIS 44(16).

\section{Author's Contributions}

All the authors contributed equally to prepare, develop and carry out this manuscript.

\section{Ethics}

Authors declare that are not ethical issues that may arise after the publication of this manuscript. This article is original and contains unpublished material.

\section{References}

Halliday, D. and R. Robert, 1966. Physics, Part II. 1st Edn., John Wiley and Sons, Inc., New York.

Harold Urey, from Wikipedia, the free encyclopedia. https://en.wikipedia.org/wiki/Harold_Urey.

Hydrogen, from Wikipedia, the free encyclopedia. https://en.wikipedia.org/wiki/Hydrogen.

Krane, K.S., 1987. Introductory Nuclear Physics. 3rd Edn., Wiley and Sons, New York, ISBN-10: 047180553X, pp: 864

Kramer, D., 2011. DOE looks again at inertial fusion as a potential clean-energy source. Phys. Today, 64: 26-26 DOI: $10.1063 / 1.3563814$

Moses, E.I., R.N. Boyd, B.A. Remington, C.J. Keane and R. Al-Ayat, 2009. The national ignition facility: Ushering in a new age for high energy density science. Phys. Plasmas, 16: 041006-041006. DOI: $10.1063 / 1.3116505$

Petrescu, F.I., 2012. Cold nuclear fusion. Plasma Physics and Fusion Technology (S70), INIS.

Petrescu, F.I. and J.K. Calautit, 2016a. About nano fusion and dynamic fusion. Am. J. Applied Sci., 13: 261-266 DOI: 10.3844/ajassp.2016.261.266

Petrescu, F.I. and J.K. Calautit, 2016b. About the light dimensions. Am. J. Applied Sci., 13: 321-325. DOI: 10.3844/ajassp.2016.321.325

Petrescu, F.I. and R.V. Petrescu, 2012. News in Physics. 1 st Edn., Books on Demand, ISBN-13: 978-3848229642, pp: 82.

Petrescu, F.I., A. Apicella, R.V. Petrescu, S. Kozaitis and R. Bucinell et al., 2016. Environmental protection through nuclear energy. Am. J. Applied Sci., 13: 941-946.

DOI: 10.3844 /ajassp.2016.941.946

"Progress in Fusion". ITER. http://www.iter.org/sci/beyonditer.

Reid, R.V., 1968. Local phenomenological nucleonnucleon potentials. Annals Phys., 50: 411-448.

Shultis, J.K. and R.E. Faw, 2002. Fundamentals of nuclear science and engineering. CRC Press, New York, ISBN-10: 0824708342, pp; 520.

Thermonuclear weapon, from Wikipedia, the free encyclopedia.

https://en.wikipedia.org/wiki/Thermonuclear_weapon 
Relly Victoria Virgil Petrescu et al. / American Journal of Engineering and Applied Sciences 2017, 10 (3): 703.708 DOI: 10.3844/ajeassp.2017.703.708

\section{Nomenclature}

$\begin{array}{lll}\varepsilon_{0} & \text { The permissive constant (the } \\ & \text { permittivity) } \\ = & 8.85418 \mathrm{E}-12\left[\mathrm{C}^{2} / \mathrm{Nm}^{2}\right] \\ = & \text { The Planck constant: } \\ \varepsilon_{0} & 6.626 \mathrm{E}-34[\mathrm{Js}] \\ h & =\text { Electrical elementary load: } \\ h & = & -1.6021 \mathrm{E}-19[\mathrm{C}] \\ q & = & 1.6021 \mathrm{E}-19[\mathrm{C}] \\ q_{e} & =\text { The light speed in vacuum: } \\ q_{p} & = & 2.997925[\mathrm{~m} / \mathrm{s}] \\ c & = \\ c & =9.11 \mathrm{E}-31[\mathrm{~kg}] \\ m_{0}[\mathrm{~kg}] & =1,6726219 \mathrm{E}-27[\mathrm{~kg}] \\ m_{0 e l e c t r o n} & =\text { Universal gravitational constant } \\ m_{0 p r o t o n} & =6,674087 \mathrm{E}-11\left[\mathrm{Nm}^{2} / \mathrm{kg}^{2}\right] \text { or }\left[\mathrm{m}^{3} / \mathrm{kg} \cdot \mathrm{s}^{2}\right] \\ G\left[\mathrm{Nm}^{2} / \mathrm{kg}^{2}\right] & \end{array}$

